\title{
Labyrinthe
}

27 | 2007 (2)

La fin des disciplines?

\section{Une question de tempérament : l'in(ter)discipline des penseurs africains-américains}

\section{Anthony Mangeon}

\section{OpenEdition}

Journals

Édition électronique

URL : https://journals.openedition.org/labyrinthe/2018

DOI : $10.4000 /$ labyrinthe. 2018

ISSN : 1950-6031

Éditeur

Hermann

Édition imprimée

Date de publication : 4 juillet 2007

Pagination : 59-75

ISBN : 978-2-9526131-4-9

Référence électronique

Anthony Mangeon, «Une question de tempérament : l'in(ter)discipline des penseurs africainsaméricains », Labyrinthe [En ligne], 27 | 2007 (2), mis en ligne le 25 mars 2011, consulté le 27 avril 2022. URL : http://journals.openedition.org/labyrinthe/2018; DOI : https://doi.org/10.4000/labyrinthe. 2018

Propriété intellectuelle 


\title{
UNE QUESTION DE TEMPERAMENT : L'IN(TER)DISCIPLINE DES PENSEURS AFRICAINS-AMÉRICAINS
}

\author{
Anthony MANGEON \\ anthony.mangeon@club-internet.fr
}

Au début du $\mathrm{XX}^{\mathrm{e}}$ siècle, l'interdisciplinarité ne préoccupait guère les universitaires des États-Unis et la société américaine, majoritairement, n'imaginait point d'autre avenir que la ségrégation dans les relations raciales. De nouveaux projets sociaux, culturels, s'élaboraient pourtant dans l'ombre, en s'appuyant audacieusement sur des recherches qui décloisonnaient les savoirs. L'initiative venait d' «intellectuels nègres » qui, en se définissant comme tels, assumaient désormais leur «double conscience» à la façon d'un paradoxisme: Américains et Noirs, Intellectuels et Nègres - fiers de cette double appartenance, mûs par de profondes exigences de rigueur et d'universalité, et forts de singuliers héritages, sans testament, échus d'une dépossession. Parmi eux, deux brillantes figures, bientôt appelées à servir de proue: William Edward Burghardt Du Bois (1868-1963) et Alain Leroy Locke (1885-1954).

Tous deux licenciés de Harvard, fleuron de la Ivy League, après une première formation ambitieuse (le premier à Fisk University, le second à la Philadelphia School of Pedagogy), Du Bois et Locke vont suivre des cheminements pluridisciplinaires et transnationaux: à l'exemple de leur mentor, le philosophe William James (1842-1910), ils vont en effet étudier en Europe, et s'inscrire notamment à l'université de Berlin, respectivement en 1892-1894 et 1910-1911. Féru de philosophie, mais prudemment pourvu d'une maîtrise d'histoire' ${ }^{1}, \mathrm{Du}$ Bois écrira sa thèse sur La Suppression de la traite des Noirs en Amérique (publiée en 1896)

\footnotetext{
1. James l'avait dissuadé d'entreprendre une carrière universitaire en tant que philosophe, qu'il jugeait en effet difficile et risquée, sinon presque impossible pour un Noir dans la société américaine de l'époque.

2. The Suppression of the African Slave-Trade to the United States of America, 1638-1870, dans Du Bois, Writings, New York, Library of America, 1996.
} 
avant d'ouvrir la voie de la sociologie urbaine avec une remarquable étude sur la condition des Noirs dans le Sud (The Philadelphia Negro, 1899). Devenu professeur d'histoire et d'économie à l'université d'Atlanta, il fera paraître en 1903 un singulier essai, The Souls of Black $F_{o l k}{ }^{1}$, où son propos retrace, entre histoire, sociologie, autobiographie et critique culturelle, l'aventure collective des Noirs américains jusqu'à l'aube du XX $\mathrm{X}^{\mathrm{e}}$ siècle. En 1905, le jeune universitaire fonde, avec vingtneuf autres «intellectuels nègres », un mouvement de lutte pour la reconnaissance des droits civiques qui donnera naissance, en 1910, à la National Association for the Advancement of Colored People (NAACP); cette même année, Alain Locke rejoint l'université de Berlin, après trois ans passés au Hertford College de l'université d'Oxford; il y rédige une première thèse de philosophie, Un essai sur le concept de valeur ${ }^{2}$, et suit, entre autres, les cours de Georg Simmel (1858-1918) avant de rejoindre Paris au printemps 1911 pour assister aux leçons d'Henri Bergson (1859-1941) au Collège de France. À son retour aux États-Unis, il est recruté par l'université d'Howard (Washington D. C.), pour y enseigner la littérature et la philosophie. Sous les auspices de la NAACP, il y délivre, en 1915 et 1916, une série de conférences publiques et interdisciplinaires (entre sociologie, histoire et anthropologie) sur Les Contacts de race et les relations interraciales ${ }^{3}$, et revient alors à Harvard pour obtenir, en 1918, son $\mathrm{PhD}$ de philosophie avec une nouvelle thèse sur la théorie des valeurs ${ }^{4}$. Quelques années plus tard, Locke édite l'anthologie du New Negro: polymorphe et polyphonique, réunissant sans distinction ni préséance des hommes et des femmes, des Noirs et des Blancs, des écrivains, des artistes, des journalistes et des chercheurs, ce volume s'offre tout à la fois comme un nouveau corpus (de poèmes, fictions et théâtre...) et comme une première collection d'essais critiques (en sociologie, en histoire, en critique littéraire, musicale ou artistique); des transcriptions de contes ou de Negro Spirituals, des reproductions d'art tradi-

\footnotetext{
1. Les Âmes du peuple noir, traduction, annotation et postface de Magali Bessone, Paris, Éditions Rue d’Ulm, 2005; rééd. La Découverte/Poche, 2007.

2. An Essay on the Concept of Value, Moorland-Spingarn Research Center, Howard University, Manuscript Division, Alain Locke Papers, boîte 164-125.

3. Race Contacts and Interracial Relations, édité et introduit par Jeffrey Stewart, Washington D. C., Howard University Press, 1992.

4. The Problem of Classification in the Theory of Value, An Outline of a Genetic System of Values, Moorland-Spingarn Research Center, Howard University, Manuscript Division, Alain Locke Papers, boîte 164-155.
} 
tionnel ou contemporain, des bibliographies et discographies complètent intelligemment l'ensemble, marquant ainsi l'entrée des «Nouveaux Nègres » sur la scène culturelle et intellectuelle américaine. Sans multiplier davantage les détails biographiques, je voudrais, en me concentrant sur certains essais de W. E. B. Du Bois et d'Alain Locke, explorer leurs manières d'unir et d'infléchir, d'altérer et de contraindre les disciplines. Dans un tel programme, tout peut s'entendre à double sens: "unir et infléchir» les disciplines, cela signifie les accorder en des lieux communs, mais cela implique également, dans le contexte où vivent et pensent les intellectuels noirs, de les détourner de leurs traditionnelles ambitions théorétiques, de leurs habituelles tentations universalistes, pour les contraindre à penser les problèmes spécifiques de l'expérience africaineaméricaine: en un mot, de tester pragmatiquement ce que William James appelait ironiquement leur «valeur au comptant ${ }^{1} »$. Quant aux «disciplines», il ne faut pas seulement les entendre comme des unités ou des découpages du savoir, mais également y reconnaître les régimes conceptuels qui les ordonnent et nous font entrer, sur le plan intellectuel, dans de promptes habitudes réflexives. Il s'agit donc, en somme, d'unir et d'infléchir des disciplines autant que les régimes conceptuels qui les traversent. Sans prétendre que ces in(ter)disciplines sont exclusivement africaines-américaines, j'espère toutefois montrer combien elles témoignent d'un processus original qui consiste, ici, à « noircir» ou à «africaniser» discours, théories et concepts pour rendre compte des situations, aspirations et expressions des peuples dominés. Comme le rappelaient dès les années trente, et sur un autre plan, Cyril Lionel Robert James (1901-1989) et Léon-Gontran Damas (1912-1978)², les droits de l'homme et du citoyen ne devinrent véritablement universels qu'avec leur réappropriation par les esclaves noirs de Saint-Domingue, qui les actualisèrent pour leur propre compte. Conséquemment, parler d'un «internationalisme noir», d'un «marxisme noir» ou de «Lumières noires» ne serait point restreindre ces idiomes politiques ou culturels à un sens

\footnotetext{
1. «La méthode pragmatiste, écrivait James en 1907, consiste à interpréter chaque notion en retraçant ses conséquences pratiques. Quelle différence pratique pourrait-on constater si telle notion s'avérait vraie plutôt que telle autre? [...] Vous devez extraire de chaque mot sa valeur pratique au comptant (practical cash-value), et la tester au cœur de votre propre expérience.» Voir «Ce que le Pragmatisme signifie», dans John McDermott (dir.), The Writings of William James, A Comprehensive Edition, Chicago, Chicago University Press, 1996, p. 380.

2. C. L. R. James, Les Jacobins noirs, 1936, Paris, Gallimard (1951 pour la traduction); L.-G. Damas, «89 et Nous, les Noirs» (Europe, $\mathrm{n}^{\circ} 193,15$ juillet 1939).
} 
particulariste, mais bien éprouver leur valeur à l'aune d'un test pragmatique, ainsi que l'entendait William James. En d'autres termes, parler ici d'une «interdisciplinarité noire» ne serait qu'une invitation à explorer plus avant la manière dont certains «intellectuels nègres » ont contribué au développement et à la compréhension des indisciplines dans la recherche.

\section{ALTERNER/ALTÉRER LES RÉGIMES DE PENSÉE}

Les critiques de la Renaissance de Harlem ont souvent mis en relief l'influence profonde qu'exerça William James sur Du Bois et Locke, qui furent tous deux ses étudiants ${ }^{1}$. En revanche, l'impact qu'eurent certains penseurs européens sur leurs réflexions et leurs écrits demeure relativement négligé; bien plus, alors même qu'on prétend souligner leurs points communs, on laisse habituellement de côté la manière dont ils infléchirent et lièrent ensemble concepts et disciplines. Je n'ambitionne pas ici une exhaustive histoire des idées: je veux seulement montrer qu'en suivant James, Simmel et d'autres penseurs américains ou européens, Du Bois et Locke semblent moins influencés par des systèmes spécifiques ou des concepts précis, que mûs et agis par les régimes de pensée antithétiques ou par les logiques antagonistes qui traversent ces systèmes et ces concepts, qu'ils cherchent à infléchir, à remodeler en retour.

Depuis Platon qui opposait, dans Le Sophiste, les «Fils de la terre» aux «Fils du ciel», le champ philosophique s'est en effet traditionnellement partagé en deux tendances, ou deux manières de se situer à l'égard $\mathrm{du}$ «plan d'immanence», cette surface que trace tout philosophe dès lors qu'il propose un discours sur et une connaissance de l'expérience 2 .

D'un côté, sur la face supérieure, la tendance idéaliste se concentre sur les capacités de l'esprit à «refléter» l'ordre des choses, et elle conçoit donc la philosophie comme une théorie de la connaissance dont le but est de «clarifier ce qui rattache le schème représentationnel dont nous nous

\footnotetext{
1. Voir George Hutchinson, The Harlem Renaissance in Black and White, Cambridge (Mass.) \& Londres, the Belknap Press of Harvard University Press, 1995; Ross Possnock, Color and Culture, Black Writers and the Making of the Modern Intellectual, Cambridge (Mass.), Harvard University Press, 1998; Mark Helbling, The Harlem Renaissance, The One and The Many, Westport (Connecticut) \& Londres, Greenwood Press, 1999.

2. Gilles Deleuze et Félix Guattari, Qu'est-ce que la philosophie?, Paris, Minuit, 1991.
} 
servons au contenu que nos représentations visent ${ }^{1} »$. En tant qu'il «donne une image à la pensée», ce noologisme, pour parler comme Deleuze et Guattari, part ainsi d'un antifondationnalisme foncier à l'égard de toutes nos croyances ordinaires, et il manifeste notamment un scepticisme radical, à l'égard tantôt de la connaissance par les sens (Descartes), tantôt de la métaphysique (Kant), tantôt de la connaissance objective elle-même (Husserl), pour mieux défendre ensuite les pouvoirs de l'esprit, détaché des contingences corporelles, et fonder alors la connaissance sur des conditions a priori de possibilité, ou des «contraintes spécifiques qu'exerce la "rationalité" et qui expliquent pourquoi nos théories idéales doivent "correspondre à la réalité" ${ }_{\text {» }}$. Motivé par la recherche de structures stables et permanentes, ce régime philosophique s'articule sur une logique fixiste - celle de l'identité et de la différence. Ses habitudes conceptuelles sont, par conséquent, le dualisme (entre la forme et le contenu, entre le sujet et l'objet, entre la psyché et le monde), la hiérarchie (ou la subordination du corps à l'âme, de l'émotion au jugement, du contenu à la forme, de l'objet au sujet, du monde à la psyché) et pour finir, le finalisme qui règle et oriente les rapports entre les deux pôles du dualisme et de la hiérarchie, selon les deux versants complémentaires d'un même effort de transcendance: on a ainsi, du point de vue interne, une visée téléologique, où la faculté supérieure (raison ou jugement) se prend elle-même pour fin, ou bien s'instaure comme aboutissement des facultés inférieures (imagination et émotion); on dispose également, du point de vue externe, d'une vision progressiste, où la croyance peut se muer en certitude et la connaissance devenir de plus en plus «adéquate», jusqu'à coïncider si possible avec le point de vue de l'absolu. Indexée sur le modèle sensoriel de la vue, la connaissance comme théorein, c'est-à-dire vision, est envisagée sur le mode rétinien de la représentation spéculaire; «comprendre comment approfondir la connaissance, c'est comprendre comment améliorer le fonctionnement d'une faculté quasi visuelle - le "miroir de la nature" -, et donc penser la connaissance comme une collection de représentations adéquates $^{3} »$, ou un «savoir de» dont l'objectivité repose sur le principe de la correspondance.

1. Richard Rorty, L'Homme spéculaire [1979], Paris, Le Seuil, 1990, p. 439 pour la traduction française.

2. Ibidem, p. 328.

3. Ibidem, p. 187. 
Face à cette option, et constituant de fait l'autre face du plan d'immanence, une tradition alternative s'est constituée en s'opposant à la tendance intellectualiste qu'elle assimile à une altération de l'ordre naturel; faisant fond sur les paradoxes du régime philosophique dominant, et motivée par la nécessité de le dépasser, elle se propose d'en renverser les habitudes conceptuelles, et d'abandonner notamment sa logique formelle, aprioriste, pour rétablir une logique génétique et naturaliste qui veut que «toute explication soit une explication causale de ce qui existe, et qu'il n'existe rien de tel qu'une condition non causale de possibilité ${ }^{\gg} \gg$. Il n'est donc plus question d'imiter les lois de l'esprit dans son fonctionnement, mais bien celles de la nature dans sa production, et il s'agit de rétablir, face au besoin de transcendance et à la quête de permanence, le primat de l'immanence et de son dynamisme. Ce régime conceptuel, que j'appellerais turbulent, a donc lui aussi son histoire, sous-jacente à l'ordre dominant du discours philosophique. Lucrèce, Spinoza ou Herder défendent par exemple la même conception naturaliste et axiologique du rapport au monde, et ils proposent une nouvelle conception de la connaissance comme «adéquation», non plus conçue comme pure représentation mais comme connaissance génétique - par les causes de production qui entraîne dès lors une nouvelle conception de la philosophie, non plus comme théorie de la connaissance, mais comme discours sur la nature et, en particulier, sur la nature humaine: en somme, une éco-anthropologie. À rebours du régime noologiste, ce régime physicaliste part, lui, d'un fondationnalisme à la fois métaphysique et empirique (Deus sive Natura chez Spinoza ou Herder, l'élan vital chez Bergson ou la métaphysique de l'expérience chez James ou Dewey), pour se découvrir sceptique à l'égard de toute prétention fondationnaliste qui ne serait pas ancrée dans la physique même des choses, et ouvrir ainsi la voie à un antifondationnalisme d'autant plus radical qu'il ne se connaît d'autre limitation que l'immanence elle-même. Ses habitudes conceptuelles sont donc le parallélisme (ou l'union intrinsèque du corps et de l'esprit, de la forme et du contenu), le relationnisme (ou l'interaction constante et constitutive du monde et de la psyché, du sujet et de l'objet, de la conscience avec d'autres consciences), et le causalisme (ou la détermination comme enchaînement de causes) qui débouchent, à travers la fluctuation constante de l'agir et

\footnotetext{
1. Richard Rorty, «Wittgenstein, Heidegger et la réification du langage», Essais sur Heidegger et autres écrits, Paris, PUF, 1991, p. 90.
} 
du pâtir, sur la complémentarité des contraires et des postulations contradictoires. Dans cette perspective, la connaissance est indexée sur le modèle sensoriel de l'ouie, son principe est la cohérence, ses attributs la continuité et l'accord, et elle est envisagée sur le mode de la mise en relation propositionnelle, c'est-à-dire, fondamentalement, comme un «savoir que».

Il importe de bien comprendre les dynamiques concurrentielles de ces deux régimes conceptuels, car c'est précisément dans leur dialectique que furent immergés les intellectuels noirs, et notamment Locke et Du Bois, durant leurs formations philosophiques et sociologiques. À Harvard, les partisans de l'idéalisme hégélien, Josiah Royce (18551916) et George Herbert Palmer (1842-1933), et les défenseurs d'un empirisme radical, William James et George Santayana (1863-1952), avaient coutume de proposer dans leurs cours des réfutations argumentées de leurs vues respectives. Plus tard, à l'université de Berlin, Max Weber (1864-1920) et Georg Simmel insisteraient à leur tour sur l'antagonisme des philosophies qui allait de pair, selon eux, avec la pluralité des systèmes de valeur dans la vie sociale, et ils argumenteraient en faveur d'une pratique philosophique qui prendrait sérieusement en compte - et tâcherait même de justifier - le relativisme et la diversité des rapports au monde, tout en maintenant, parallèlement, la quête de schèmes structuraux et communs de la pensée. Ainsi Weber allait-il forger, d'un côté, des «idéaux-types » (ou concepts abstraits à même de saisir les phénomènes empiriques et les relations concrètes, tout en demeurant des idéaux qui excèdent les aspects de l'expérience qu'ils aident à penser); et de l'autre, Simmel se concentrerait quant à lui sur les processus dynamiques de «l'action réciproque» (Wechselwirkung) dans la vie sociale, sans pour autant négliger l'exploration de ce qu'il appelait les «formes $a$ priori de la socialisation ${ }^{1} »$. À l'instar de Weber et Simmel, et dans la filiation de James qui avait d'abord opposé, dans son Pragmatisme, deux tempéraments philosophiques antithétiques, les «esprits durs» et les «esprits mous», pour tenter d'opérer entre eux une médiation dans ses conférences sur Philosophie de l'expérience: un univers pluraliste $(1908)^{2}$, Du Bois et Locke se trouvèrent rapidement tentés de dépasser

\footnotetext{
1. Voir l'anthologie de ses essais parue sous le titre Sociologie, études sur les formes de socialisation, Paris, PUF, 1999.

2. Traduction française par David Lapoujade, Paris, Les Empêcheurs de penser en rond, 2007.
} 
le traditionnel antagonisme des logiques conceptuelles pour se situer dans une sorte d' «entre-deux» lorsqu'ils commencèrent à explorer les relations possibles entre, d'une part, l'éthique et l'esthétique et, d'autre part, les idées de race et de culture.

L'origine et l'antagonisme des valeurs, l'articulation entre les caractéristiques raciales et les traits culturels constituaient de fait des sujets majeurs des débats philosophiques au tournant des XIX et $\mathrm{XX}^{\mathrm{e}}$ siècles, et les penseurs africains-américains ne pouvaient y rester indifférents, même si leurs voix n'étaient pas les plus entendues. Je ne m'étendrai pas ici sur les essais de $\mathrm{Du}$ Bois sur «La renaissance de l'éthique» et «La conservation des races »: d'autres l'ont fait brillamment ${ }^{1}$. Il nous suffit de constater que ses spéculations philosophiques furent reconduites et approfondies, dans une certaine mesure, par Alain Locke, qui s'avéra en outre bien plus persévérant que Du Bois dans son exploration d'une possible «science des valeurs», et tout aussi engagé que lui dans la compréhension du concept de race. Locke, on l'a vu, écrivit en effet deux thèses de doctorat sur la théorie des valeurs, outre divers articles qu'il publia dans des volumes collectifs ${ }^{2}$; il produisit par ailleurs, durant sa carrière académique, de nombreuses essais sur les problèmes sociaux et les développements historiques des relations interraciales, aussi bien aux ÉtatsUnis qu'en Europe ou dans les Antilles: outre ses conférences de 19151916, déjà mentionnées, il convient de citer également, dans les années 1940, ses conférences en français sur Le Rôle du Nègre dans la culture des Amériques ${ }^{3}$, précédées de plusieurs coordinations scientifiques sur ce sujet (un numéro spécial du Survey Graphic, intitulé Color, the Unfinished Business of Democracy, en 1942, et, la même année, une anthologie de textes historiques, anthropologiques et sociologiques : When Peoples Meet, A Study in Race and Culture Contacts ${ }^{4}$ ). En raison même de leur finesse argumentative, ses écrits sur la valeur, la race et la culture présentent encore aujourd'hui un grand intérêt.

\footnotetext{
1. Voir notamment Anthony Appiah, In My Father's House, New York, Oxford University Press, 1992; David Levering Lewis, W. E. B. Du Bois, Biography of a Race, 1868-1919, New York, Henry Holt and Co, 1994, et la postface de Magali Bessone à sa traduction des Âmes du peuple noir.

2. Rassemblés dans Leonard Harris (dir.), The Philosophy of Alain Locke, Harlem Renaissance and Beyond, Philadelphie, Temple University Press, 1989.

3. Port-au-Prince, Imprimerie de l'État, 1943; rééd. L'Harmattan, coll. "Autrement Mêmes», Paris, 2007 (à paraître).

4. New York, Progressive Education Association, 1942.
} 
Locke conçut en effet précisément sa théorie des valeurs comme une médiation entre les deux régimes conceptuels dont je viens de retracer l'antagonisme. C'est, par exemple, grâce à la psychologie naturaliste de William James (lequel insiste sur la dimension première, affective de la conscience, et fait ainsi du sentiment un véritable mode de connaissance) que Locke tempère la psychologie intellectualiste de Franz Brentano, lequel mettait plutôt l'accent sur la dimension secondaire, réflexive de la conscience, et privilégiait ainsi l'introspection comme seul mode de connaissance adéquate, en rapportant notamment l'origine et le fondement véritable des jugements de valeurs à un rapport intra-mental de la conscience à ses sensations et perceptions ${ }^{1}$. Inversement, c'est grâce à l'idéalisme d'Heinrich Rickert, qui constate l'autonomie relative de l'esprit vis-à-vis du corps et défend, par conséquent, l'indépendance des sciences de la culture envers les sciences de la nature, que Locke parvient à tempérer, dans la théorie des valeurs, le physiologisme d'un Nietszche ou le biologisme intégral d'un Robert Eisler' ${ }^{2}$. Mais dans les deux cas, la stratégie spéculative est bien la même, qui induit une médiation et poursuit une conciliation entre les deux régimes conceptuels antagonistes: d'un côté, Locke désamorce la tentation d'une échappée dans le point de vue transcendantal, et refuse ainsi de rapporter nos valeurs à une simple conformité avec des catégories logiques (la définition du bien, du beau, du vrai, du juste, etc.); mais, dans le même temps, il refoule la tentation d'un physiologisme radical, et refuse de rapporter la constitution et la diversité des valeurs à de simples échelles hédonistes ou à des distinctions physiologiques et behavioristes. Le projet philosophique d'Alain Locke consiste donc à remettre en question la traditionnelle partition entre la valorisation, qui relève du sentiment, et l'évaluation, qui relève du jugement. Il s'attache à montrer, d'une part, comment la valorisation rend possible le jugement de valeur, c'est-à-dire en quoi elle est ellemême, déjà, du jugement; il tâche d'exposer, d'autre part, comment le jugement de valeur prolonge la valorisation, c'est-à-dire en quoi il reste

1. Psychologie du point de vue empirique, traduction de l'allemand et préface de Maurice de Gandillac, Paris, Aubier, 1944.

2. Voir Heinrich Rickert, Science de la culture et science de la nature, traduit de l'allemand par AnneHélène Nicolas, Caroline Prompsy et Marc de Launay, Paris, Gallimard, 1997; Friedrich Nietzsche, Par-delà Bien et Mal et La Généalogie de la morale, Paris, Gallimard, coll. «Folio Essais », 1987 \& 1989; Robert Eisler, Studien zur Werttheorie, Leipzig, Duncker \& Humblot, 1902. 
lui-même du sentiment. Nous avons là un exemple caractéristique de cette stratégie de médiation qui consiste à alterner et altérer les régimes conceptuels: contre le régime dominant de la pensée occidentale, les intellectuels noirs tendront souvent à favoriser le régime turbulent, mais ils refuseront dans le même temps de s'y confiner, car cela reviendrait alors à favoriser un monologisme aux dépens d'un autre. Ils ne le mettront donc pas tant en opposition qu'en dialogue avec le premier, cherchant à se situer en somme dans leurs plis, leurs insterstices, leurs intervalles. D'une certaine manière, Locke inaugure ici ce que d'autres penseurs indisciplinés, tels Mikhail Bakhtine (1895-1975) et Gregory Bateson (1904-1980), poursuivront eux aussi au cours du XXe siècle; il introduit aussi, entre les régimes conceptuels, cette logique du don et sa règle de réciprocité que Mauss découvrait au fondement des sociétés «archaïques», et dont en sa qualité première d'anthropologue, Bateson constatait la faillite dans les schèmes relationnels privilégiés en Occident ${ }^{1}$. Dans sa volonté de trouver une médiation entre l'intentionnalité de Brentano et le naturalisme de James, le projet lockien d'une science des valeurs se situe en outre à la même croisée des chemins que la phénoménologie de Husserl, mais il chemine à l'opposé puisqu'il refuse de faire de l'intentionnalité une condition a priori du savoir. Tous les efforts de Husserl s'apparentaient à une tentative presque désespérée de récupérer, au bénéfice du régime dominant, les acquis conceptuels du régime turbulent, installé au cœur de l'immanence, pour les mettre au crédit du point de vue transcendantal; mais Locke, comme Bergson, choisit inversement de maintenir la primauté du «flux de l'expérience» dont procède, en dernier ressort, toute formalisation: pour eux, la dimension première de la conscience, c'est la temporalité, qui seule permet une structuration de l'expérience: «Conscience signifie d'abord mémoire», disait Bergson, et ce qu'il importe dès lors de «ressaisir», c'est bien «la durée et le changement dans leur mobilité originelle ${ }^{2} »$, ainsi que les cristallisations de la mémoire en vue de la pratique. La véritable science de la conscience, c'est donc la théorie des valeurs, et le véritable problème de

1. Marcel Mauss, «Essai sur le Don» [1924], dans Sociologie et anthropologie, Paris, PUF, coll. «Quadrige», 2003; Gregory Bateson, «Contact culturel et schismogenèse» [1935], Vers une écologie de l'esprit, t. I, Paris, Le Seuil, coll. «Points», 1995.

2. Henri Bergson, «La Conscience et la vie», L'Énergie spirituelle, dans Euvres, Paris, PUF, 1959, p. 818 . 
la philosophie, c'est celui de l'acquisition, du développement et des mutations des modes structurants de la valorisation. Pour spécifier la genèse de ces sentiments dispositionnels, Locke s'inspirera d'un autre philosophe et psychologue français, Théodule Ribot (1839-1916), à qui il emprunte les notions oxymoriques d' «abstraits émotionnels» et de «jugements affectifs» pour opérer sa médiation entre les deux régimes conceptuels, et soutenir ainsi l'idée que les types ou les jugements de valeur, dans leurs fréquents antagonismes, doivent être rapportés à des modes affectifs différenciés, dans la valorisation ${ }^{1}$. Tandis qu'il est toujours possible d' 'optimiser un mode de valorisation lui-même, quel qu'il soit, en tant qu'attitude et activité ${ }^{»}$, Locke plaidait également pour une prise de conscience de la réciprocité et de la complémentarité nécessaire des divers modes de valorisation dans l'expérience humaine. La théorie lockienne des valeurs constituait donc bien une alternative complète et novatrice à la phénoménologie transcendantale. Elle ne cherchait nullement, en effet, à promouvoir une énième version de la philosophie-entant-que-théorie-de-la-connaissance, - mais défendait plutôt une anthropologie pragmatiste qui œuvrait pour la transformation du champ culturel; elle n'établissait point, par ailleurs - et à rebours de Husserl -, que l'Europe et ses valeurs constituaient «quelque chose d'un genre unique, que tous les autres groupes humains eux-mêmes ressentent, et qui est pour eux [...] une incitation à s'européaniser toujours davantage ${ }^{3} »$, mais soutenait davantage la promesse qu'à partir de nos divers modes de valorisation, fussent-ils esthétiques, éthiques, religieux, logiques, etc., nous puissions précisément nous arracher à cet ethnocentrisme foncier, quel que soit notre niveau de culture.

Si nous prêtons à présent quelque attention à sa théorie de la race, Locke nous paraît déployer exactement la même stratégie d'alternance et d'altération réciproque des régimes de pensée. Dans le gobinisme ${ }^{4}$, le spencerisme social et les autres théories racistes qui dominaient à l'époque

\footnotetext{
1. Voir Théodule Ribot, La Logique des sentiments, 1905, rééd. L’Harmattan, Paris, 2000.

2. «Values and Imperatives», p. 38, The Philosophy of Alain Locke, op. cit.

3. Edmund Husserl, «La Crise de l'humanité européenne et la philosophie», La Crise des sciences européennes et la phénoménologie transcendantale, traduit de l'allemand et préfacé par Gérard Granel, Paris, Gallimard, 1976, p. 353-354.

4. Voir Arthur de Gobineau, Essai sur l'inégalité des races humaines, 2 vol., Paris, Firmin-Didot, 1863-1865.
} 
l'anthropologie sociale ou la sociologie, Locke n'avait guère de peine à reconnaître l'empreinte du régime dominant dont la logique réifiante, l'obsession pour la permanence et la hiérarchie interféraient avec le régime turbulent, son affirmation d'un strict parallélisme entre le corps et l'esprit, ou sa préoccupation pour les processus d'interaction réciproque dans la nature et la société. Face aux préjugés racistes et pseudo-scientifiques, des anthropologues comme Franz Boas (1858-1942) ou Melville Herskovits (1895-1963) tâchaient de débarrasser le naturalisme des thèses erronées de l'invariabilité et de la hiérarchie des types humains, et ils étudiaient notamment, avec les outils de l'anthropométrie, les importants changements survenus, d'une génération à l'autre, chez les immigrés ou les Noirs des États-Unis. De leurs démonstrations, ils concluaient alors, dans la pure logique du parallélisme, qu'en raison de changements physiques si manifestes, des changements mentaux et des développements intellectuels se trouvaient conséquemment tout aussi possibles que plausibles ${ }^{1}$. Tout en s'ancrant lui-même dans le régime turbulent, avec ses préoccupations génétiques et son insistance sur l'action réciproque, Locke adopta une stratégie bien différente, puisqu'à rebours de Boas il ne soutint pas un strict parallélisme entre corps et esprit, mais argumenta pour une coupure fondamentale entre les traits physiologiques et les aptitudes mentales, psychologiques, intellectuelles: là encore, il se trouvait fidèle aux enseignements d'Henri Bergson qui avait défendu, dans Matière et Mémoire (1896), un nécessaire dualisme entre le corps et l'esprit. Mais le penseur noir alla plus loin encore: tandis que Boas et Herskovits spécifiaient, à travers des mesures anthropométriques, quelles différences raciales existaient d'un groupe ou d'une génération à l'autre, Locke visait surtout à déterminer les sources, non plus des différences ou des inégalités, mais des iniquités et des discriminations dans la vie sociale. Et c'est ici que l'infléchissement des régimes de pensée se combine avec celui des disciplines.

\footnotetext{
1. Franz Boas, «Changes in Immigrant Body Form», «Instability of Human Types», «The Outlook for the American Negro», dans George W. Stocking, Jr. (dir.), The Shaping of American Anthropology. A Franz Boas Reader, Chicago, University of Chicago Press, 1974; Melville Herskovits, «The Racial Hysteria», Opportunity, juin 1924; «Preliminary Observations in a Study of Negro-White Crossing», Opportunity, mars 1925. Pour l'anecdote, Opportunity était une « revue de la vie nègre», et le dernier texte de Boas («Outlook...») est en réalité la conférence qu'il délivra à Atlanta University en 1906, à l'invitation de Du Bois.
} 
Une question de tempérament : l' in(ter)discipline des penseurs...

\section{CONJOINDRE/CONTRAINDRE LES DISCIPLINES}

Dans la théorie des valeurs, le questionnement philosophique rencontrait la réalité sociale, et débouchait ainsi sur une problématisation sociologique. Locke empruntait ainsi certaines vues à Georg Simmel, Émile Durkheim (1858-1917) et Gabriel Tarde (1843-1904): sa description d'un mode de valorisation s'apparente à la conception simmélienne d'un Denkmodus, tandis que ses analyses de «l'empathie», de «l'influence sociale» et du «conditionnement de la valorisation par d'autres qui interagissent avec ou contre elle» font converger la préoccupation structurale de Durkheim pour les présuppositions sociales avec l'insistance génétique de Tarde sur Les Lois de l' imitation ${ }^{1}$.

Quand Locke aborde la théorie de la race, c'est en revanche la question sociale qui devient un problème philosophique, ou plutôt: c'est un problème social - la discrimination raciale - qui va se découvrir également des racines conceptuelles. L'anthropologie et la sociologie sont d'abord mobilisées à nouveau pour expliquer les préjugés et les formes d'interaction (domination et subordination, ségrégation, conflit, croisement des cercles sociaux...). Ses lectures sociologiques, d'inspiration marxienne et tardienne, permettent à Locke d'expliquer les pratiques et les théories raciales en les rapportant à leurs causes historiques, matérialistes et psychologiques: les hiérarchies raciales apparaissent toujours, dans l'histoire, comme des cristallisations de statuts différenciés, mais également comme la rationalisation d'une volonté de pouvoir ${ }^{2}$; par conséquent, ce n'est ni la démonstration scientifique des errements du racisme, ni la dialectique matérialiste de la lutte des classes qui suffiront à éradiquer les pratiques et les théories raciales. Puisque le sentiment racial trouve sa source dans le sentiment de «parenté» (kinship feeling), «base anthropologique de toute organisation sociale», « les classes économiques peuvent bien être absorbées, nos castes psychologiques n'en seront pas dissoutes pour autant ${ }^{3} »$. Nous retrouvons ici cette double stratégie de «maîtrise de la forme» et «déformation de la maîtrise» que j’ai

\footnotetext{
1. An Essay on the Concept of Value, p. 131; The Problem of Classification..., p. 129. Émile Durkheim, «Représentations individuelles et représentations collectives», dans Philosophie et Psychologie, Paris, PUF, 1974.

2. Race Contacts and Interracial Relations, p. 11-12.

3. «Values and Imperatives», dans Leonard Harris (dir.), The Philosophy of Alain Locke. Harlem Renaissance and Beyond, Philadelphie, Temple University Press, 1989.
} 
déjà explicitée dans les pages de cette revue ${ }^{1}$. «Maîtriser les formes» signifie comprendre quelles logiques intellectuelles et culturelles soustendent les logiques sociales et politiques; et pour cela, la maîtrise des sciences humaines et sociales permet d'expliciter les causes historiques de la discrimination sociale, et aussi d'exposer les ambiguïtés du sentiment d'appartenance, qui, dans un état d'esprit culturaliste, peut fonctionner de manière positive et active, mais également donner naissance à des pratiques racistes et réactionnaires ${ }^{2}$. Cette stratégie n'a toutefois d'autre fin que de permettre une déformation des rapports traditionnels de domination entre Européens et non-Européens, Blancs et Noirs américains. Déformer cette «maîtrise» implique donc, parallèlement, de déconstruire l'hégémonie du régime dominant en révélant ses paradoxes et contradictions. Tarde, dans ses Lois de l'imitation (1895), et Simmel, avec sa sociologie des conflits (Soziologie der Konkurrenz), ont montré que des frontières sociales étanches, des systèmes ségrégationnistes ne font qu'exacerber le désir mimétique des groupes dominés; par ailleurs, l'exploitation sexuelle des femmes noires, le métissage qui en résulte, et la réalité du passing - ou passage, d'un groupe racial à l'autre, des individus suffisamment clairs de peau - non seulement contredisent le dogme de la pureté raciale, sa hantise de la dégénérescence, mais sapent également la possibilité même du biracialisme à moyen terme ${ }^{3}$. Pour contrer cette logique sociale moniste et hégémonique, Locke préconise donc en retour la systématisation des dynamiques sociales. L'imitation ne saurait se limiter, pour lui, à la contrefaçon des groupes dominants par ceux qui leur sont subordonnés, dans la mesure où toute interaction sociale implique une influence symétrique - quoique souvent passée sous silence - des derniers sur les premiers: mentor du mouvement Nouveau Nègre, Locke multipliera les efforts pour remplacer l'arbitraire «ligne de couleur» par «une double ligne d'influences interactives entre la majorité et la minorité, lesquelles se déterminent réciproquement et se modifient l'une l'autre ${ }^{4} »$. Ses essais soutiendront tout particulièrement

\footnotetext{
1. Voir mon texte «Maîtrise et déformation, les Lumières diffractées» dans Labyrinthe, ${ }^{\circ}$ 24, 2006, p. 63-83.

2. Voir Race Contacts..., p. 20, et l'essai «Jingo, Counter-Jingo and Us», dans Jeffrey Stewart (dir.), The Critical Temper of Alain Locke, A Selection of His Essays on Art and Culture, New York \& Londres, Garland Publishing Inc, 1983, p. 258-259.

3. Le Rôle du Nègre dans la culture des Amériques, p. 75 et p. 82 ; When Peoples Meet, p. 237.

4. C'est en ces termes qu'il présentera en 1949 l'objectif visé par son anthologie The New Negro. An Interpretation («Wisdom de Profundis »), dans Jeffrey Stewart (dir.), The Critical Temper..., op. cit., p. 358).
} 
l'imitation des formes d'expression populaire et toutes les dynamiques d'invention comme «entrecroisement» de différents processus d'imitation, base de toute créativité humaine selon Tarde ${ }^{1}$. Mais par-delà cette systématisation des dialogismes sociaux, la déformation des rapports traditionnels de maîtrise s'obtiendra également en transformant la loyauté - dont Simmel faisait une forme a priori de la socialisation - en principe d'action: Locke propose en effet de favoriser ce schème relationnel pour contrer l'esprit partisan, et il reproduit ainsi le geste téléologique de l'idéalisme, où la structure intentionnelle se prend elle-même pour fin, en empruntant à Josiah Royce son principe de «loyalisme au loyalisme» qu'il indexe, quant à lui, sur une préoccupation toute pragmatiste pour ses effets dans l'expérience sociale. «Dans le choix et dans le service de la cause que vous devez servir loyalement, soyez, en toute occurrence, loyal envers le loyalisme ${ }^{2} »$, recommandait Royce; cette devise, Locke l'appliquera toujours en conformité avec sa conception oxymorique de l'identité, entre universalité et singularité, puisque dans sa défense et illustration du nationalisme culturel noir, il tâchera constamment de rester fidèle au cosmopolitanisme et à l'américanisme .

\section{MODE MINEUR}

Pour conclure, je voudrais risquer une analogie: telle qu'elle fut pratiquée par Locke, mais aussi par d'autres penseurs africains, caribéens et

\footnotetext{
1. «L'art soi-disant cultivé peut bien se mettre à l'école de l'art populaire, et apprendre ainsi, à refondre et reformer sa matière, pour produire de nouvelles œuvres qui sont la synthèse de l'art académique et de l'art naïf, de la culture populaire et de la culture cosmopolite. C'est donc un véritable mariage, et non point une mésalliance, entre ces éléments que proposent nos artistes et nos penseurs d'avant-garde», dans Le Rôle du Nègre dans la culture des Amériques, p. 24.

2. Josiah Royce, Philosophie du loyalisme [1908], traduction française de Jacqueline Morot-Sir, Paris, Aubier, 1946, p. 75.

3. Dès 1909, Locke écrit, dans l'épilogue de la revue Cosmopolitan, qu'il avait contribué à créer à Oxford: «La culture cosmopolite, dès lors qu'on peut vraiment la cultiver, c'est avoir le sentiment des contrastes de valeurs, et un recentrement sur soi accru et rationalisé... », dans Cosmopolitan, I, 1909, p. 16); quinze ans plus tard, son introduction à l'anthologie du New Negro se conclut ainsi : «L'esprit du Nègre n'aspire à rien d'autre qu'aux désirs américains, aux idées américaines. Mais cette tentative (qu'on lui impose) de construire son américanisme sur des valeurs propres à sa race est une expérience sociale unique, et le succès est en dernier ressort impossible si l'on se refuse à lui faire intégralement partager la culture et les institutions américaines », dans The New Negro [1925]; New York, Simon \& Schuster, 1992, p. 12.
} 
africains-américains, ainsi que je l'ai exposé ailleurs ${ }^{1}$, l'interdisciplinarité s'apparenterait, en termes musicaux, à une question de tempérament. L'accord d'un instrument consiste en effet à faire résonner les harmoniques de deux notes pour trouver un juste milieu entre le dièse de l'une et le bémol de celle qui lui est immédiatement supérieure, et l'interdisciplinarité pourrait elle-même consister à superposer et infléchir les régimes de pensées, à lier et altérer les disciplines pour s'en servir à l'amble. Mais je voudrais ajouter qu'il se joue une expérimentation particulière dans les versions africaines-américaines de l'interdisciplinarité. À l'instar du Blues, où le tempérament africain, en tant qu'organisation pentatonique, resurgit au sein même de l'échelle des notes européenne, en «tordant» (bending) et en «aplanissant » (flattening) les troisième et septième majeures, l'interdisciplinarité, telle qu'elle fut pratiquée par les premiers penseurs africains-américains, impliquait aussi de «noircir» et d'«africaniser» les théories, les concepts et les pratiques pour leur permettre d'exprimer leur expérience propre. C'est ainsi, par exemple, que William Du Bois emprunte simultanément ses concepts à William James et Georg Simmel, pour caractériser sa «double conscience» et son sentiment d'invisibilité, comme derrière un «voile»:

Le Noir est [...] né avec un voile et doué de double vue dans ce monde américain [...]. C'est une sensation bizarre, cette conscience dédoublée, ce sentiment de constamment se regarder par les yeux d'un autre. [...] Chacun sent constamment sa nature double - un Américain, un Noir; deux âmes, deux pensées, deux luttes irréconciliables ; deux idéaux en guerre dans un seul corps noir, que seule sa force inébranlable prévient de la déchirure. L'histoire du Noir américain est l'histoire de cette lutte - de cette aspiration à être un homme conscient de lui-même, de cette volonté de fondre son moi double en un seul moi meilleur et plus vrai².

\footnotetext{
1. Notamment dans une thèse de littérature générale et comparée: Lumières noires, Discours marron: indiscipline et transformations du savoir chez les écrivains noirs américains et africains, université de Cergy-Pontoise, 2004.

2. Les Âmes du peuple noir, p. 11. Du Bois s'inspire, dans cet autoportrait du Nègre, d'un article de James, «The Hidden Self» (Essays in Psychology, 1890), où ce dernier avait systématisé les observations de Pierre Janet, à partir de séances d'hypnose, pour établir la possibilité que chacun d'entre nous ait différentes personnalités situées à des niveaux distincts de la conscience: James distinguait ainsi une conscience primaire d'une conscience secondaire, qui possédaient chacune des attributs sensoriels, affectifs et mémoriels propres, qui ne pouvaient se rejoindre qu'à un troisième niveau de conscience, plus profondément enfoui. Quant à Simmel, il avait précisément théorisé l'importance du
} 
Ce processus d'appropriation, il nous faut donc l'envisager aussi dans sa dimension réciproque, et bien nous inspirer en retour des contributions nègres au projet interdisciplinaire. Mélange unique de pratiques européennes et africaines, le Blues, de l'avis même de Sterling Brown, grand poète des Nouveaux Nègres, est à l'origine d'une tonalité particulière - musicale, mais aussi affective («deep melancholy, in spite of some humor») -, et il s'ancre ainsi dans une expérience singulière pour mieux s'ouvrir au partage universel: «Ne vous êtes-vous jamais trouvé avec l'esprit qui divague en mille directions opposées? Une partie de vous en allée, et l'autre qui reste ancrée ${ }^{1}$ ?»

À sa semblance, l'interdisciplinarité peut se concevoir comme un savoir sans frontières, mais aussi sans illusions: en somme un nouveau «gai savoir», né du désenchantement et du décentrement des humanités.

regard d'autrui dans les relations sociales en forgeant la métaphore du voile: «Nous sommes tous des fragments », expliquait-il, « non seulement de l'autre, mais de nous-mêmes. [...] Mais le regard de l'autre complète cet aspect fragmentaire pour former ce que nous ne sommes jamais totalement et uniquement. [...] La base vitale commune donne naissance à certaines hypothèses à travers lesquelles on se voit mutuellement comme à travers un voile. Bien sûr, la singularité de la personne n'est pas purement et simplement voilée, mais dans la mesure où son existence tout à fait individuelle et concrète se fond avec cet a priori en une unité, ce voile lui donne une forme nouvelle. [...] Partout la généralisation sociale voile les traits de la réalité», dans «Le Problème de la sociologie», Sociologie, op . cit., p. 69-70. Une fois appropriés et «noircis» par Du Bois, ces concepts de double conscience et de voile serviront à caractériser plus généralement les situations ou les effets de l'oppression, et demeurent encore très (trop?) utilisés aujourd'hui dans les théories postcoloniales.

1. "What are the Blues?», conférence à la Bibliothèque du Congrès en compagnie d'Alain Locke, à l'occasion d'un concert qui célébrait le soixante-quinzième anniversaire de l'Abolition américaine de l'esclavage (disponible en compact-disc: Freedom!, New York, Bridge Records Inc., 2002, n 9114). 